\title{
Characteristics and processing of Pol IV transcripts in Arabidopsis
}

\author{
Hsuan Yu Kuo ${ }^{\mathrm{a}, 1}$, Elise L. Jacobsen ${ }^{\mathrm{a}, 1}$, Yanping Long ${ }^{\mathrm{b}}$, Xinyuan Chen ${ }^{\mathrm{a}}$ and Jixian \\ Zhai $^{\text {ba, }, *}$
}

${ }^{a}$ Department of Molecular, Cell and Developmental Biology, University of California at Los Angeles, Los Angeles, CA 90095, USA;

${ }^{\mathrm{b}}$ Department of Biology, South University of Science and Technology, Shenzhen 518055, China

${ }^{1}$ These authors contributed equally to this work.

*Correspondence: zhaijx@sustc.edu.cn (J.Zhai)

In plants, RNA-directed DNA methylation (RdDM) plays an essential role in silencing transposable elements that would otherwise have a deleterious effect on genome integrity. RdDM is an important pathway that establishes and maintains de novo DNA methylation in all three sequence contexts: $\mathrm{CG}, \mathrm{CHG}$, and $\mathrm{CHH}$ (where $\mathrm{H}$ is $\mathrm{A}, \mathrm{C}$, or T), and the methylation is targeted by 24-nt small interfering RNAs (siRNAs) (Law and Jacobsen, 2010). A plant-specific RNA polymerase (Pol IV) is required to initiate the production of 24-nt siRNAs by transcribing single-stranded RNA precursors at target loci (Law and Jacobsen, 2010; Matzke and Mosher, 2014). The precursors serve as templates for RNA-dependent RNA polymerase 2 (RDR2) to make double-stranded RNAs (dsRNAs), which are then cleaved by Dicer-like 3 (DCL3) into 24-nt siRNAs (Haag et al., 2012). Many studies were conducted to explore the biogenesis of 24-nt siRNAs; however, the nature of Pol IV precursor RNAs was unclear due to the rapid conversion into siRNAs (Li et al., 2015). 
Here, we focus on recent studies that have greatly improved our understanding of the characteristics of Pol IV-dependent transcripts. We first start by characterizing the nature of Pol IV-dependent RNAs (P4RNAs) as well as insights into the biogenesis and termination of P4RNAs prior to their conversion into 24-nt siRNAs (Blevins et al., 2015; Li et al., 2015; Zhai et al., 2015). We then follow up with discussion of other reports regarding the processing of P4RNAs in a dicer-independent manner (Yang et al., 2016; Ye et al., 2016). For each section, we will discuss recent advances regarding P4RNAs as well as alternative models and interpretations.

\section{Identity of Pol IV RNAs}

Until recently, the assumed pathway for the biogenesis of 24-nt siRNAs begins with Pol IV transcribing long single-stranded precursor RNAs that are converted into dsRNAs by RDR2 and cleaved by DCL3 to form 24-nt siRNAs (Law and Jacobsen, 2010; Mosher et al., 2008). The structure of these precursor RNAs have been difficult to characterize due to the transient nature of the transcripts prior to dicer processing as well as the low transcription level at silent Pol IV loci (Li et al., 2015; Zhai et al., 2015). Li et al. (2015) were the first to identify regions generating Pol IV transcription, and their findings suggest that the transcripts (P4RNAs) are in the range of 100-500-nt based on clustering of adjacent reads, which supports the traditional model where processing of long Pol IV precursor RNAs produce multiple siRNAs. However, several more recent studies, based on the size of individual transcripts, suggest that P4RNAs are actually less than 50-nt in length and each proceeds to become to a single 24-nt siRNA (Blevins et al., 
2015; ; Zhai et al., 2015; Yang et al., 2016). Since Pol IV evolved from RNA polymerase II (Pol II) ( Ream et al., 2009; Blevins et al., 2015), P4RNAs could theoretically possess similar features as Pol II transcripts. On the contrary, it appears that instead of having a 7methylguanosine cap that is characteristic of Pol II transcripts, P4RNAs have a 5' monophosphate (Li et al., 2015; Zhai et al., 2015). Furthermore, Li et al. (2015) found that P4RNAs lack introns and are not polyadenylated, which suggests that P4RNAs are distinct from Pol II transcripts (Li et al., 2015). In the field of RNAi, there has been the notion that misprocessing of transcripts by RDR proteins could lead to the generation of aberrant RNAs that trigger silencing (Herr et al., 2006). However, recent data have shown that P4RNAs are not simply long and misprocessed siRNAs. These studies characterized P4RNAs in $d c l 2-1 d c l 3-1$ dcl4-2 triple mutant background since dicer proteins can act redundantly with one another, and the precursor RNAs were shown to dramatically accumulate in $d c l 2 / 3 / 4$ compared to the wild type (Li et al., 2015; Zhai et al., 2015). In contrast, the level of Pol IV siRNAs showed drastic reduction in $d c l 2 / 3 / 4$ compared to the wild type, which suggests that P4RNAs are produced upstream of dicers and are the immediate precursors of 24-nt siRNAs (Li et al., 2015; Zhai et al., 2015). Current studies also indicate that P4RNAs and siRNAs show overlap with annotated transposable elements (TEs) and are enriched at the borders of long TEs (Li et al., 2015; Zhai et al., 2015), providing further support for the direct connection. Although siRNAs exist as a duplex, it has been shown that AGO4 selectively binds to one strand that serves as the guide for the downstream targeting of RdDM (Zhong et al., 2014). Consistent with this model, emerging studies illustrate that P4RNAs and siRNAs can be strand specific and map to the same strand, which suggests that the Pol IV-derived strand most 
frequently becomes the final 24-nt siRNA products (Yang et al., 2016; Zhai et al., 2015) that target DNA methylation.

\section{Initiation of Pol IV RNAs}

The canonical model of RdDM posits that siRNA biogenesis begins with Pol IV occupying heterochromatic regions and actively transcribing precursor RNAs, which are then processed by RDR2 (Law and Jacobsen, 2010). Although this may be the case, it has been shown that Pol IV could not produce P4RNAs without the presence of RDR2 (Blevins et al., 2015; Li et al., 2015; Zhai et al., 2015). To further support the coupling of activities of Pol IV and RDR2, the precursor RNAs that dramatically accumulated in $d c l 2 / 3 / 4$ mutants become depleted if either of the two proteins is absent (Blevins et al., 2015; Li et al., 2015; Zhai et al., 2015). Li et al. (2015) suggested that initiation of Pol IV transcription occurred in A/T-rich and nucleosome depleted regions. However, their method did not detect the actual orientation of P4RNA reads due to the double-stranded nature of the Pol IV/RDR2 products. Later analyses on 5' ends of P4RNAs illustrated that there was a strong enrichment of $\mathrm{T} / \mathrm{C}(\mathrm{Y})$ at the -1 position and $\mathrm{A} / \mathrm{G}(\mathrm{R})$ at the +1 position, which indicates that Pol IV follows the Y/R rule and initiates transcription in a similar manner as Pol II (Blevins et al., 2015; Zhai et al., 2015). In addition, the 5' enrichment for purine (A or G) in P4RNAs is consistent with the finding that DCL3 prefers short dsRNAs that contain a 5' adenine (Nagano et al., 2014). Most detected P4RNAs are shorter than 45-nt, and together with the finding that DCL3 cleaves preferentially at a fixed distance from $5^{\prime}$ adenine, on average only one 24-nt siRNA duplex will be generated from each precursor (Blevins et al., 2015; Zhai et al., 2015). In 
short, Pol IV initiates transcription at sites that follow the Y/R features, and together with RDR2, generates short transcripts enriched for 5' purine in order to facilitate DCL3 processing.

\section{Termination of Pol IV RNAs and $3^{\prime}$ mismatches}

Further sequence composition analysis revealed that P4RNA reads with a perfect match to the genome showed enrichment for A, C, U at the last three nucleotides of the $3^{\prime}$ end (Blevins et al., 2015; Zhai et al., 2015). A recent study extended the investigation of $3^{\prime}$ nucleotide preference in order to determine if there was a difference between P4RNAs that start with 5' A or 5' G. The sequence logos illustrate that while all P4RNAs preferentially end with $U$, the order of preference differs in that 5'A P4RNAs prefer $U$ > A > C whereas 5' G P4RNAs prefer $\mathrm{U}>\mathrm{C}>\mathrm{A}$ (Blevins et al., 2015). In addition, 24-nt siRNAs show a nucleotide preference of $\mathrm{U}>\mathrm{C}>\mathrm{A}$ at the $3^{\prime}$ end as well. This suggests a model in which DCL3 processes 5' A P4RNAs from the 5' end and 5' G P4RNAs from the $3^{\prime}$ end (Blevins et al., 2015).

When allowing for multiple mismatches in the genome while mapping P4RNAs, an interesting phenomenon arises. The mismatches are mainly localized to the $3^{\prime}$ ends of P4RNAs and consist of one or two nucleotides that do not match the expected sequence

(Blevins et al., 2015; Zhai et al., 2015; Yang et al., 2016). A comparison of P4RNAs with perfect genome match and those with mismatches showed that while the $5^{\prime}$ end base composition was very similar between the two, the $3^{\prime}$ ends illustrated a large variation in base preference (Zhai et al., 2015). This finding suggests that while Pol IV initiates transcription in a stereotypical manner, there might be different termination mechanisms 
at play (Zhai et al., 2015). An in vitro experiment demonstrated that recombinant RDR2 can catalyze the addition of one or two non-templated cytosines to the $3^{\prime}$ end of RNA, suggesting that RDR2 has terminal transferase activity that could account for the $3^{\prime}$ mismatches observed in Pol IV/RDR2 RNAs (Blevins et al., 2015).

Alternative to the model where RDR2 adds non-templated nucleotides, Zhai et al. (2015) noted that there were different preferences for non-templated nucleotides that were dependent on the sequence of the DNA template, which suggests that the termination of P4RNAs with mismatches results from misincorporation during Pol IV transcription. Furthermore, the misincorporation occurred most often at positions where guanines were expected, which would correspond to cytosines on the DNA template (Zhai et al., 2015). Upon further analysis, CG dinucleotides showed the highest enrichment out of all dinucleotide combinations and were strongly enriched at the $3^{\prime}$ ends of P4RNAs with misincorporation (Zhai et al., 2015). Taking into account the trend where CG dinucleotides in Arabidopsis have the highest level of methylation (Cokus et al., 2008; Lister et al., 2008) and the regions in the genome producing Pol IV siRNAs are usually heavily methylated at CG sites (Stroud et al., 2013), it appears that cytosine DNA methylation is contributing in part to the $3^{\prime}$ misincorporation of P4RNAs (Zhai et al., 2015). Further supporting the role of DNA methylation in the misincorporation was the finding that $\mathrm{CG}$ dinucleotide enrichment was significantly reduced in $d d m 1$ (decrease in DNA methylation1) $d c l 3$ double mutant compared to $d c l 3$ single mutant, where $d d m 1$ mutant exhibited a significant loss of methylation but was still producing 24-nt siRNAs (Zhai et al., 2015). By terminating P4RNAs at sites of pre-existing DNA methylation, a self-reinforcing loop would be created in which the resulting siRNAs direct DNA 
methylation targeting and DNA methylation in turn helps to pinpoint the location for further siRNA production (Zhai et al., 2015).

\section{Pol IV RNAs at dicer-independent loci}

A recent study extended the role of P4RNAs at dicer-independent RdDM loci. In the $d c l 1 / 2 / 3 / 4$ mutant, 24-nt siRNAs theoretically should be depleted due to a lack of processing by dicers, resulting in a reduction of DNA methylation at RdDM loci. However, according to the report of Yang et al. (2016), there are many RdDM loci that show a near wild-type level of DNA methylation in the absence of all four DCL proteins, and only $16 \%$ of loci fully rely on dicers. As a result of this disconnection, they proposed that at many RdDM loci, 24-nt siRNAs are not required for DNA methylation. To further characterize this anomaly, Yang et al. (2016) categorized RdDM loci into dicer fullydependent (FD), partially-dependent (PD), and independent (ID) loci, and they found that euchromatin marks (H3K4me3, H3K4me2, H3K36me2, H3K36me3, H3K9Ac, H3K18Ac) were generally associated with FD loci and heterochromatin marks (H3K9me2, H3K27me1) were associated with PD and ID loci (Yang et al., 2016). Furthermore, at PD and ID loci, P4RNAs increased in accumulation in $d c l 1 / 2 / 3 / 4$ mutant compared to $d c l 2 / 3 / 4$ mutant, and this trend correlated with higher DNA methylation (Yang et al., 2016). This finding suggests that P4RNAs function as trigger RNAs for directing DNA methylation at initial stages of RdDM (Yang et al., 2016).

Another study explored the role of siRNAs that are independent of DCLs (sidRNAs). The P4siRNAs that accumulated in $d c l 1 / 2 / 3 / 4$ mutant are mostly 18-30-nt long, have a 5' monophosphate, and require functional NRPD1 (largest subunit of Pol IV) 
and RDR2 (Ye et al., 2016). However, they did not observe a difference in the level of sidRNA production in $d c l 2 / 3 / 4$ and $d c l 1 / 2 / 3 / 4$ mutants. Moreover, Ye et al. (2016) proposed that biogenesis of sidRNAs is also dependent on AGO4, as sidRNA level in $d c l 2 / 3 / 4$ mutant reduced dramatically with mutation in ago4. In addition, the transgenic expression of wild-type AGO4 was able to rescue the depletion of sidRNAs in ago4 dcl2/3/4 whereas mutant AGO4 defective in small RNA (sRNA) binding was not, indicating the importance of sRNA binding activity of AGO4 for sidRNA generation (Ye et al., 2016). From their AGO4 immunoprecipitation, Ye et al. (2016) observed that AGO4 bound sidRNAs were smaller in size compared to the sidRNAs detected in $d c l 2 / 3 / 4$ mutant and suggested that the longer forms are precursors of the mature sidRNAs that associate with AGO4. Their proposed model is that after $5^{\prime}$ binding of precursor sidRNAs to AGO4, a 3'-5' exonuclease trims the transcripts to form mature sidRNAs that function as initial triggers of RdDM (Ye et al., 2016).

\section{Concluding remarks}

Recent evidence has shed light on the nature of Pol IV dependent transcripts. A large consensus of these studies indicates that the transcripts are in the range of 30-50-nt in length, have a 5' monophosphate, require the coupling activity of Pol IV and RDR2, and show a strong overlap with 24-nt siRNAs. These P4RNAs largely accumulate in $d c l 2 / 3 / 4$ mutants, which are accompanied by the dramatic reduction of 24-nt siRNAs, suggesting that P4RNAs are the immediate precursors of 24-nt siRNAs. Some of these studies performed sequence composition analysis on P4RNAs and found enrichment for 5' purine $(\mathrm{A} / \mathrm{G})$, which corresponded to previous findings that showed DCL3 to 
preferentially process dsRNAs with 5'A as well as AGO4 loading preference for 24-nt siRNAs with 5'A. The sequence composition analysis also revealed that P4RNAs were enriched for 3' U although the order preference of nucleotides varied slightly between studies. In addition, the analyses revealed that P4RNAs were enriched for $\mathrm{Y}$ at -1 position and $\mathrm{R}$ at +1 position, indicating that Pol IV initiates transcription at positions that resemble the Y/R features of Pol II transcription start sites (TSSs). Lastly, many of the recent findings showed non-templated nucleotides at the $3^{\prime}$ ends of P4RNAs; however, each proposed a different theory to explain the phenomenon.

A few studies proposed an alternative pathway for the Pol IV dependent transcripts that appear to act independently of dicers. Yang et al. (2016) and Ye et al. (2016) performed analyses in $d c l 1 / 2 / 3 / 4$ mutants, which suggests that Pol IV dependent transcripts are capable of directing DNA methylation in absence of 24-nt siRNAs. However, DCL1 is a hypomorphic allele and $d c l 1$ null mutants show embryonic lethality; thus, it is still possible that the remnants of DCL proteins in the $d c l 1 / 2 / 3 / 4$ mutants might be sufficient in low levels of siRNA production to trigger DNA methylation. On the other hand, Ye et al. (2016) argued that although miRNA accumulation reduced drastically in $d c l 1 / 2 / 3 / 4$ relative to $d c l 2 / 3 / 4$ mutants, siRNAs generated from P4RNA loci remained at comparable levels, suggesting that they may not be produced by the residual activity of DCL1 in $d c l 1 / 2 / 3 / 4$ mutant. In the future, it would be recommended to use conditional null mutants of $D C L 1$ in certain cell types to more definitively study dicer independence and the resulting effects on RdDM.

Despite the increase in our understanding of Pol IV dependent transcripts, the key question of how Pol IV terminates transcription remains unknown. Although a few of the 
studies found 3' U enrichment in P4RNA reads that matched perfectly to the genome, it is unclear as to whether Pol IV terminates transcription at a specific sequence or if it transcribes a longer sequence that is then processed by some uncharacterized endonuclease. Lastly, the mechanistic understanding of the manner in which P4RNAs incorporate $3^{\prime}$ mismatches is lacking and requires further investigation.

\section{Acknowledgments}

We thank Javier Gallego-Bartolome and other members of the Jacobsen lab for insightful discussions. H.Y.K. was supported by a grant from the Bill \& Melinda Gates Foundation to the Jacobsen lab. J.Z. was a Life Science Research Foundation postdoctoral fellow, sponsored by the Gordon and Betty Moore Foundation. Group of J.Z. was supported by the Thousand Talents Program for Young Scholars and South University of Science and Technology.

\section{Figure Legends}

\section{Figure 1. Generation and processing of Pol IV dependent transcripts in Arabidopsis.}

Li et al. (2015) reported that Pol IV transcription takes place in A/T rich and nucleosome depleted regions, where long dsRNA transcripts are generated and are processed by DCL3 to form multiple 24-nt siRNAs. The other four reports found that Pol IV dependent transcripts are only 30-50 nt long and have roles via both a DCL-dependent and a DCL-independent manner. In the DCL-dependent pathway, Pol IV transcription begins at sites that follow the "Y/R" rule. Zhai et al. (2015) reported that the resulting P4RNAs are highly enriched for 5' adenine, 
which undergo a single cleavage event by DCL3 to form 24-nt siRNAs. Blevins et al. (2015) reported similar findings, but they found equal enrichment for $5^{\prime}$ adenine and 5' guanine in P4RNA reads and suggested that DCL3 processes the dsRNAs equally from both ends. In DCL-independent manner, Ye et al. (2016) suggested that Pol II and RDR6 generate sidRNA precursors that associate with AGO4 and undergo trimming to form mature sidRNAs that can initiate RdDM. Yang et al. (2016) also postulated that in $d c l 1 / 2 / 3 / 4$ mutant, P4RNAs initiate DNA methylation in absence of siRNAs at some loci. 


\section{References}

Blevins, T., Podicheti, R., Mishra, V., Marasco, M., Tang, H., and Pikaard, C.S. (2015). Identification of Pol IV and RDR2-dependent precursors of $24 \mathrm{nt}$ siRNAs guiding de novo DNA methylation in Arabidopsis. eLife 4, e09591.

Cokus, S.J., Feng, S., Zhang, X., Chen, Z., Merriman, B., Haudenschild, C.D., Pradhan, S., Nelson, S.F., Pellegrini, M., and Jacobsen, S.E. (2008). Shotgun bisulphite sequencing of the Arabidopsis genome reveals DNA methylation patterning. Nature 452, 215-219.

Haag, J.R., Ream, T.S., Marasco, M., Nicora, C.D., Norbeck, A.D., Pasa-Tolic, L., and Pikaard, C.S. (2012). In vitro transcription activities of Pol IV, Pol V, and RDR2 reveal coupling of Pol IV and RDR2 for dsRNA synthesis in plant RNA silencing. Mol. Cell 48, 811-818.

Herr, A.J., Molnar, A., Jones, A., and Baulcombe, D.C. (2006). Defective RNA processing enhances RNA silencing and influences flowering of Arabidopsis. Proc. Natl. Acad. Sci. U. S. A. 103, 14994-15001.

Law, J.A., and Jacobsen, S.E. (2010). Establishing, maintaining and modifying DNA methylation patterns in plants and animals. Nat. Rev. Genet. 11, 204-220.

Li, S., Vandivier, L.E., Tu, B., Gao, L., Won, S.Y., Li, S., Zheng, B., Gregory, B.D., and Chen, X. (2015). Detection of Pol IV/RDR2-dependent transcripts at the genomic scale in Arabidopsis reveals features and regulation of siRNA biogenesis. Genome Res. 25, 235245.

Lister, R., O'Malley, R.C., Tonti-Filippini, J., Gregory, B.D., Berry, C.C., Millar, A.H., and Ecker, J.R. (2008). Highly integrated single-base resolution maps of the epigenome in Arabidopsis. Cell 133, 523-536.

Matzke, M.A., and Mosher, R.A. (2014). RNA-directed DNA methylation: an epigenetic pathway of increasing complexity. Nat. Rev. 15, 394-408.

Mosher, R.A., Schwach, F., Studholme, D., and Baulcombe, D.C. (2008). PolIVb influences RNA-directed DNA methylation independently of its role in siRNA biogenesis. Proc. Natl. Acad. Sci. U. S. A. 105, 3145-3150.

Nagano, H., Fukudome, A., Hiraguri, A., Moriyama, H., and Fukuhara, T. (2014). Distinct substrate specificities of Arabidopsis DCL3 and DCL4. Nucleic Acids Res. 42, 1845-1856.

Ream, T.S., Haag, J.R., Wierzbicki, A.T., Nicora, C.D., Norbeck, A.D., Zhu, J.K., Hagen, G., Guilfoyle, T.J., Pasa-Tolic, L., and Pikaard, C.S. (2009). Subunit compositions of the RNA-silencing enzymes Pol IV and Pol V reveal their origins as specialized forms of RNA polymerase II. Mol. Cell 33, 192-203. 
Stroud, H., Greenberg, M.V., Feng, S., Bernatavichute, Y.V., and Jacobsen, S.E. (2013). Comprehensive analysis of silencing mutants reveals complex regulation of the Arabidopsis methylome. Cell 152, 352-364.

Yang, D.L., Zhang, G., Tang, K., Li, J., Yang, L., Huang, H., Zhang, H., and Zhu, J.K. (2016). Dicer-independent RNA-directed DNA methylation in Arabidopsis. Cell Res. 26, 66-82.

Ye, R., Chen, Z., Lian, B., Rowley, M.J., Xia, N., Chai, J., Li, Y., He, X.J., Wierzbicki, A.T., and Qi, Y. (2016). A Dicer-independent route for biogenesis of siRNAs that direct DNA methylation in Arabidopsis. Mol. Cell 61, 222-235.

Zhai, J., Bischof, S., Wang, H., Feng, S., Lee, T.F., Teng, C., Chen, X., Park, S.Y., Liu, L., Gallego-Bartolome, J., Liu, W' Henderson, I.R', Meyers, B.C, Ausin, I', Jacobsen, S.E. (2015). A one precursor one siRNA model for Pol IV-dependent siRNA biogenesis. Cell 163, 445-455.

Zhong, X., Du, J., Hale, C.J., Gallego-Bartolome, J., Feng, S., Vashisht, A.A., Chory, J., Wohlschlegel, J.A., Patel, D.J., and Jacobsen, S.E. (2014). Molecular mechanism of action of plant DRM de novo DNA methyltransferases. Cell 157, 1050-1060. 